\title{
Hipotiroidismo e insuficiencia cardiaca
}

\section{Reporte de un caso}

\author{
Prieto-Macías J*, Ponce-Márquez TA**, Arellano-Sotelo HA ***
}

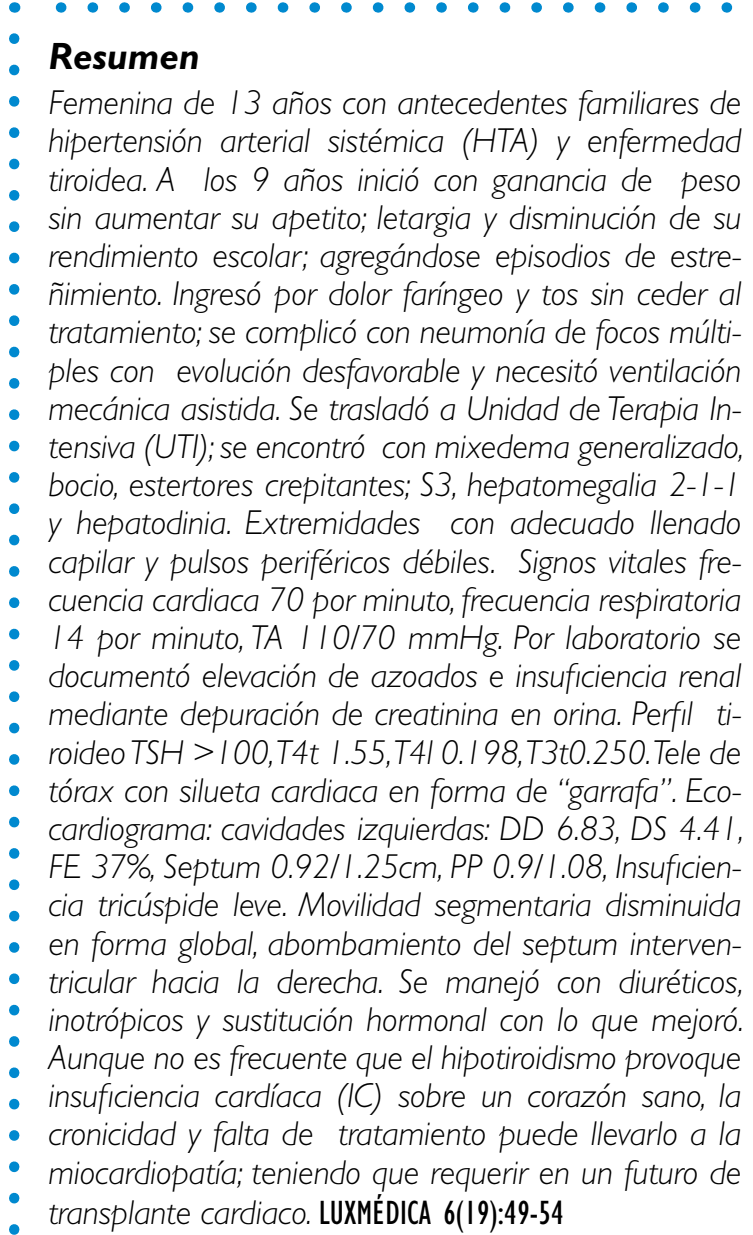

\section{Abstract}

Female 13 years of age. A family history of hypertension and thyroid disease. At the age of 9 starts with weight gain without increasing your appetite; lethargy and decreased school performance; plus episodes of constipation. Enter by pharyngeal pain and cough without yielding to treatment; It is complicated to multiple foci with unfavourable developments pneumonia requiring mechanical ventilation. Moved to UTI meets widespread myxedema, crackling, goiter, rales; S3, liver growth 2-I-I and liver pain. Limbs with appropriate filled capillary and weak peripheral pulse. Vital signs FC 70', FR 14, TA I I0/70 mmHg. Laboratory is documented elevation of azoados and renal failure through clearance of creatinine in urine profile thyroid TSH $>100$, T4t 1.55, T41 0.1 98, T3t0.250. Cabinet with Tele thorax with silhouette heart shaped "carafe" suggestive of spill. ECO. Dilated left cavities: 6.83 DD, DS 4.41, EF 37\% Septum $0.92 / 1.25 \mathrm{~cm}$, PP 0.9/1.08 IT mild. Segmental mobility decreased globally, warpage of the interventricular septum to the right. Management with diuretics, inotropes and hormone replacement to improve sastisfactory Although rare hypothyroidism cause heart failure on a healthy heart; chronicity and lack of treatment can lead to cardiomyopathy; having to require a heart transplant in the future. LUXMÉDICA 6(19):49-54

Palabras clave: hipotiroidismo, insuficiencia cardiaca.

* Profesor Investigador del Centro de Ciencias de la Salud UAA, Adscrito a la UTI del HGZ \#1 IMSS

** Médico Interno de Pregrado HGZ \#1 IMSS, Aguascalientes, Ags

*** Médico Residente de Medicina Interna de primer año HGZ \#2 IMSS; Irapuato, Gto.

Fecha de recibido 15 agosto 2011

Fecha de aceptación 30 agosto 2011

Correspondencia: Dr Jorge Prieto Macías. Departamento de Medicina. Universidad Autónoma e Aguascalientes. Avenida Universidad 940. CP 20100. Teléfono 9108436 correo electrónico dr_prieto@yahoo.com 


\section{Introducción}

Los desórdenes tiroideos frecuentemente presentan síntomas inespecíficos en los niños. ${ }^{1}$ El hipotiroidismo puede ser manifestado desde el nacimiento o posteriormente después de un periodo de normalidad de la función tiroidea. ${ }^{2}$ El desorden puede ser adquirido o puede aparecer como resultado de una variedad de defectos congénitos. ${ }^{3,4}$ La prevalencia ocurre entre el 1 al 3\% de la población en general; el congénito es de 1 por cada 5000 recién nacidos vivos. La causa más común de hipotiroidismo adquirido es la tiroiditis linfocítica crónica (TLC) y puede ser parte de un síndrome poliglandular..$^{5,6}$ La insuficiencia cardiaca aguda en la población infantil con hipotiroidismo es infrecuente y se presenta con más prevalencia cuando en la cronicidad los enfermos han desarrollado miocardiopatía dilatada. ${ }^{7}$ Debemos destacar que el hipotiroidismo no diagnosticado puede llevar a insuficiencia cardiaca y ser estos síntomas los datos más sobresalientes en su presentación inicial. .-11 $^{-11}$

Entre las complicaciones cardiacas, la miocardiopatía dilatada es la más común, con una incidencia de 0.4 a 8.0 casos por cada 100, 000 niños. Se caracteriza por alteración en la contractibilidad y dilatación de las cavidades cardiacas; histológicamente hay hipertrofia de los miocitos, fibrosis en grado variable e infiltración de matriz extra celular. ${ }^{7}$ A medida que empeora la función ventricular, incrementa la congestión y los pacientes manifiestan datos de bajo gasto. El electrocardiograma (ECG) se caracteriza por bradicardia, complejos de bajo voltaje, desviación del eje hacia la izquierda y prolongada sístole eléctrica. ${ }^{5,7}$ Ross y Reithman ${ }^{12}$ elaboraron un score clínico para valorar la severidad de la miocardiopatía que va de 0- 12 puntos, se clasifica en leve de 0-4, moderada 5-8 y severa de 9-12 puntos.

\begin{tabular}{|c|c|c|c|}
\hline & 0 puntos & 1 punto & 2 puntos \\
\hline Diaforesis & Solo en cabeza & Cabeza y cuerpo en ejercicio & Cabeza y cuerpo en reposo \\
\hline Taquipnea & Raro & Varias veces & Frecuente \\
\hline \multirow[t]{2}{*}{ Patrón respiratorio } & Normal & Retracciones & Distress \\
\hline & \multicolumn{3}{|c|}{ Frecuencia Respiratoria (rpm): } \\
\hline$<1$ Año & $<50$ & $50-60$ & $>60$ \\
\hline 1-6 años & $<35$ & $35-45$ & $>45$ \\
\hline 7-10 años & $<25$ & $25-35$ & $>35$ \\
\hline 11-14 años & $<18$ & $18-28$ & $>28$ \\
\hline \multirow[t]{2}{*}{ Hepatomegalia } & $<2 \mathrm{~cm}$ & $2-3 \mathrm{~cm}$ & $>3 \mathrm{~cm}$ \\
\hline & \multicolumn{3}{|c|}{ Frecuencia Cardiaca( Ipm) } \\
\hline$<1$ año & $<160$ & $160-170$ & $>170$ \\
\hline 1-6 años & $<105$ & $105-115$ & $>115$ \\
\hline 7-10 años & $<90$ & $90-100$ & $>100$ \\
\hline $11-14$ años & $<60$ & $80-90$ & $>90$ \\
\hline
\end{tabular}

Tabla 1. Score clínico modificado de Ross y Reithman para pacientes menores de 14 años 


\section{Presentación del caso clínico}

Femenina de 13 años de edad originaria y residente de la ciudad de Aguascalientes; fue el producto de la tercera gesta y de un embarazo sin complicaciones. Como antecedente familiar solo reveló: abuela materna con hipertensión arterial sistémica y tía con enfermedad tiroidea. Entre los antecedentes personales: los familiares refirieron que a los 9 años comenzó a ganar peso sin aumentar su apetito acompañándose de letargia y disminución de su rendimiento escolar. Un año previo a su ingreso tuvo episodios de estreñimiento. Dos semanas previas al ingreso refirió dolor faríngeo y tos productiva y se le diagnosticó faringitis. Se le dio tratamiento, sin mejoría; por lo que fue llevada al hospital donde se le diagnosticó: neumonía de focos múltiples; presentó una evolución tórpida y se agregó dificultad respiratoria muy severa que requirió intubación endotraqueal, ventilación mecánica asistida e ingreso en UTI donde se encontró: estuporosa y con edema generalizado. FC 70', FR 14', TA $110 / 70 \mathrm{mmHg}$. En cuello se documentó bocio; en tórax estertores crepitantes, en precordio S3 con ritmo de galope. Abdomen con estrías y globoso a expensas de panículo adiposo, hepatomegalia $2-1-1 \mathrm{~cm}$ y hepatodinia. Extremidades con aumento de volumen por edema, adecuado llenado capilar y pulsos débiles

\section{Exámenes de laboratorio}

Glucosa $104 \mathrm{mg} / \mathrm{dl}$, urea $50.20 \mathrm{mg} / \mathrm{dl}$, creatinina $1.6 \mathrm{mg} / \mathrm{dl}$, Ca $9.1 \mathrm{mg} / \mathrm{dl}, \mathrm{K} 3.1$ $\mathrm{mEq} / \mathrm{l}$, Na $140 \mathrm{mEq} / \mathrm{l}$, volumen urinario en $24 \mathrm{hrs}$. 5140, Urea 123, BUN 2952.09, Creatinina urinaria 16.50, Depuración de Creatinina de $39.26 \mathrm{ml} / \mathrm{min}$.

Perfil Tiroideo: TSH >100 mU/L, T4t 1: 0.55 ug/100 ml, T4L: 0.198 ng/100 ml, T3t : $0.250 \mathrm{ng} / 100 \mathrm{ml}$.

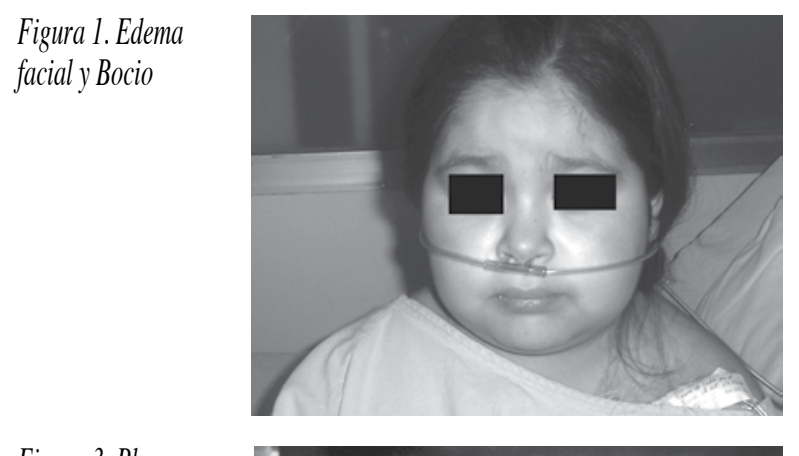

Figura 3. Placa de Tórax muestra condensación bilateral con congestión venocapilar pulmonar y silueta cardiaca en aumentada con "imagen de garrafa"

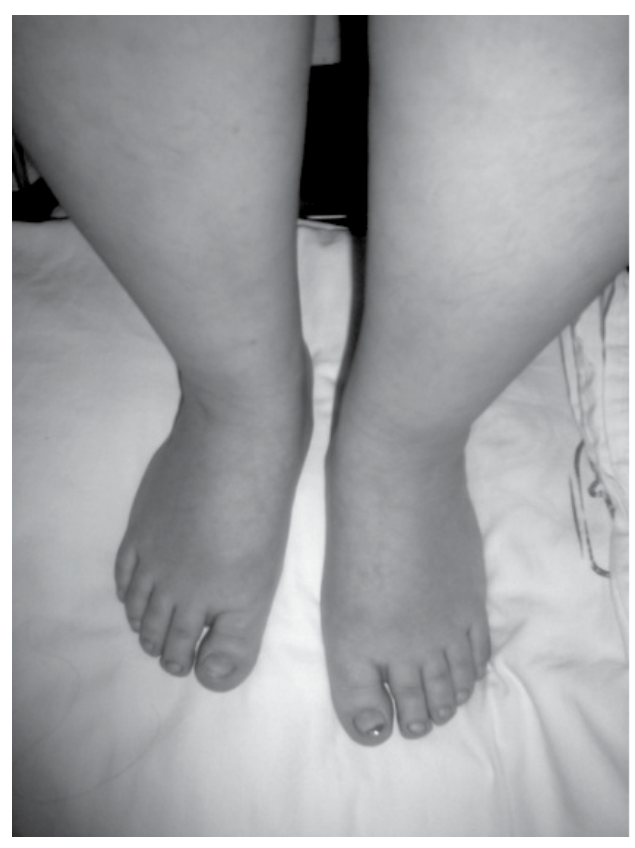

Figura 2. Edema de extremidades inferiores 


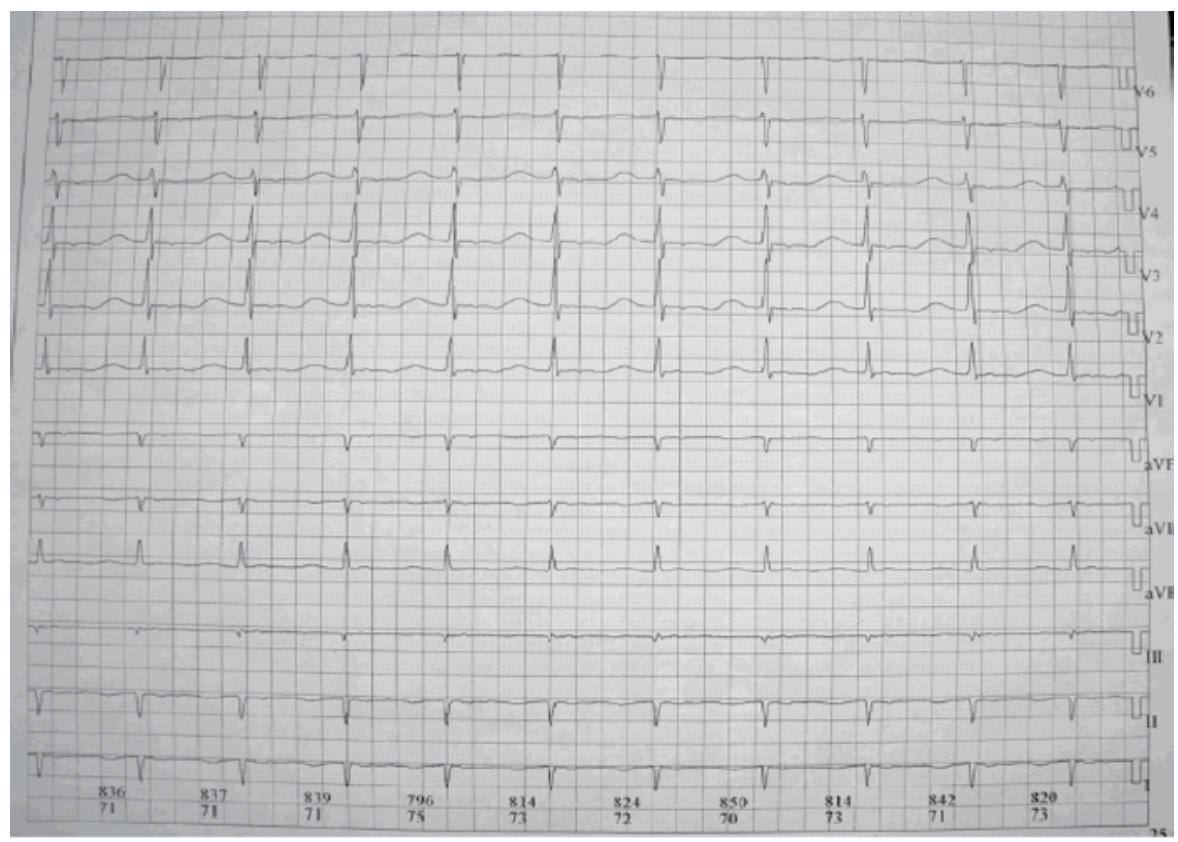

Figura 4. ECG: ritmo sinusal, AqRs $60^{\circ}$ y trastorno difuso de la repolarización

\begin{tabular}{|c|c|}
\hline & ECOCARDIOGRAMA \\
\hline DD & $6.83 \mathrm{~cm}$ \\
\hline DS & $5.41 \mathrm{~cm}$ \\
\hline $\mathrm{FE}$ & $37 \%$ \\
\hline SIV & $0.92 / 1.25 \mathrm{~mm}$ \\
\hline PP & $0.9 / 1.08 \mathrm{~mm}$ \\
\hline MITRAL & INSUFICIENCIA GRADO III \\
\hline TRICUSPIDE & INSUFICIENCIA GRADO I \\
\hline AORTICA & INSUFICIENCIA GRADO I \\
\hline MOVILIDAD & HIPOCINESIA GENERALIZADA \\
\hline DERRAME PERICARDICO & ESCASO \\
\hline
\end{tabular}

Con la terapia instituida a base de diuréticos, inotrópicos y hormonas tiroideas mejoró y fue dada de alta.

\section{Discusión}

Se presenta el caso de una niña de 13 años; con el antecedente que desde los nueve años inició con ganancia de peso, letargia y constipación; ingresó por datos de dificultad respiratoria y de insuficiencia cardiaca; con exámenes de laboratorio y de gabinete se integró síndrome de insuficiencia cardiaca e hipotiroidismo primario lo cual también puede ser la explicación de los datos de dificultad respiratoria. Con la terapéutica establecida mejoró y se dio su alta. Los cambios en el ecocardiograma se relacionan a una posible miocardiopatía dilatada con dilatación de cavidades, hipocinesia generalizada y una fracción de eyección muy disminuida. El hipotiroidismo 
per se, es una causa rara de insuficiencia cardíaca siendo más común cuando existe ya una cardiopatía de fondo; porque el índice cardíaco es usualmente suficiente para las bajas demandas del oxígeno periférico, los efectos del hipotiroidismo en el corazón son más difíciles de caracterizar con respecto a la insuficiencia cardiaca (IC)..$^{13-16}$ Es característico en la progresión de la IC que se refleje por elongamiento del miocito, con lo que se genera una dilatación de cavidades, en consecuencia, esto puede ser la mayor evidencia de que el hipotiroidismo puede eventualmente llevar a IC. Probablemente, la reducción en la masa cardíaca sea secundaria al menor trabajo miocárdico. A pesar de la reducida carga de trabajo que existente sobre el corazón hipotiroideo, la reducción del inotropismo y la frecuencia cardíaca, el aumento de las resistencias periféricas y el llenado ventricular anómalo pueden condicionar la aparición de una miocardiopatía dilatada. Dichos trastornos pueden revertir paulatinamente tras el tratamiento con hormonas tiroideas. En una reciente revisión de 673 casos de miocardiopatía dilatada se encontró que sólo dos de ellos eran debidos a hipotiroidismo. No obstante, la aparición creciente de publicaciones en las que el hipotiroidismo se presenta en forma atípica o con grave deterioro hemodinámico, hace conveniente considerar dicha patología en todo caso de insuficiencia cardíaca refractaria al tratamiento convencional. ${ }^{17-20}$

\section{Conclusiones}

La IC es infrecuente en el hipotiroidismo primario, la cronicidad de la enfermedad sin tratamiento conlleva a la expresión de bajo gasto y pude evolucionar a miocardiopatía dilatada lo que también es muy poco frecuente. En pacientes en edad infantil con inicio de insuficiencia cardiaca se deberá descartar esta posible entidad; el estudio eco-cardiográfico es uno de los elementos diagnósticos de integración. El deterioro de la función cardiaco y sistémica; aunque suele tener una evolución favorable al tratar la etiología de base (hipotiroidismo) mediante tratamiento hormonal sustitutivo; varios autores refieren que en algunos pacientes puede llegarse a requerir trasplante cardiaco por la resistencia al tratamiento.

\section{Bibliografía}

1. Moshang, Pediatric Endocrinology: The requisites in pediatrics, 1st ed. Elsevier Mosby, first edition 2005. Printed in the United States of America ISNB 0-32301825-4

2. Lafranchi sh. Thyroiditis and acquired hypothyroidism. Pediatr ann. 1992;21:29, 32-39. Kliegman: Nelson Textbook of Pediatrics, 18th ed. 2007 Saunders Elsevier ISNB: 978-1-4160-2450-7. 3200 pages
3. Victoria L. Vetter Pediatric Cardiology: requisites, 1st ed. copyright (c) 2006 Mosby Elsevier ISBN-13: 9780323023672384 pages

4. Irwin Klein And Sara Danzi .Thyroid Disease And The Heart .Circulation 2007;116;1725-1735

5. Miguel García Martínez; Ignacio Rodrigo Jarque; María Pilar Zuazola Martínez .La Insuficiencia Cardíaca Como Primera Manifestación Del Hipotiroidismo Primario Familiar.Rev Esp Cardiol. 1997;50:670772.

6. Myung K. Park, Md Pediatric Cardiology For Practitioners Fifth Edition Mosby Elsevier 2008 ISNB: 978-0-323-04636-7 

Function In Hypothyroid Children: Effect Of ReplaHeart Circulation 2007:116:1725-1735 care unit in children - Heart Fail Clin - 01-OCT-

failure in infants. Pediatr Cardiol 1992;13:72 in pa-
14. Wilson G.R. Thyroid disorders. Clinics in Family Practice. 2002; 4 (3): 65-7

15. Di Bello V., Monzani F. Ultrasonic myocardial textura analisis in subclinical hypothyroidism. Journal American Society of Echocardiography. 2000; 13:832840.

16. Bengell F., Nekolla S. Effect of thyroid hormones on cardiac function, geometry and oxidative metabolism assessed noninvasively by positron emission tomography and magnetic resonance imaging. Journal of Clinical endocrinology and Metabolism. 2000; 85(5): 1822-1827.

17. Klein, I. Thyroid hormone and cardiovascular system. American Journal of Medicine. 1990; 88: 631-637.

18. Polikar, R., Burger, A., et al. The thyroid and the heart. Circulation. 1993; 87(5). 1435-1441.

19. Biondi, B., Palmieri, E. et al. Effects of subclinical thyroid dysfunction on the heart. Annals of Internal Medicine. 2002; 137(11): 904-914.

20. www.secyt.fcm.unc.edu.ar/.../145-Hipotiroidismoe-insuficiencia-cardiaca (reporte de un caso) Juliana Salas Segura 